Check for updates

Cite this: Sens. Diagn., 2022, 1, 198

\title{
A ratiometric luminescence nanoprobe based on layered terbium hydroxide nanosheets for quantitative detection of an anthrax biomarker $\dagger$
}

\author{
Jinyan Li, ${ }^{\text {ab }}$ Qingyang Gu, (D)*ab Hui Heng, ${ }^{\text {ab }}$ Ziwei Wang, ${ }^{\text {ab }}$ \\ Haibo Jin ${ }^{\text {ab }}$ and Jing $\mathrm{He}$ (iD ${ }^{\mathrm{c}}$
}

\begin{abstract}
A novel ratiometric luminescence nanoprobe based on layered terbium hydroxide ( $L T b H)$ nanosheets was constructed for quantitative detection of dipicolinic acid (DPA), which was an anthrax biomarker. The LTbH nanosheets can be obtained by the exfoliation of the dodecyl sulfate (SDS) intercalated LTbH in formamide (FM). Upon the addition of DPA, the intrinsic green luminescence of $\mathrm{Tb}^{3+}$ can be sensitized due to the antenna effect. The luminescence intensity of FM at $332 \mathrm{~nm}$ as a reference remained almost constant, leading to ratiometric luminescence response toward DPA. The prepared luminescence nanoprobe was able to detect DPA with a linear relationship in the wide range of 1-100 $\mu \mathrm{M}$, and the detection limit was $36.67 \mathrm{nM}$. In addition, the probe showed high selectivity and sensitivity for the detection of DPA as an anthrax biomarker and can be applied in actual tap water samples.
\end{abstract}

Received 28th September 2021 Accepted 8th November 2021

DOI: $10.1039 / \mathrm{d} 1 \mathrm{sd} 00027 \mathrm{f}$

rsc.li/sensors detection is increasingly favored by researchers for its outstanding advantages such as high sensitivity, rapid response time, and simplicity. ${ }^{3}$ However, most of the currently reported DPA detection methods cannot simultaneously achieve high sensitivity and a wide linear range for DPA detection. ${ }^{3,5,6,8}$ Therefore, it is very necessary to construct a new detection system for simple, fast, sensitive, and wide-range detection of DPA.

Lanthanide ions have been explored as excellent fluorescence probe materials due to their unique spectral properties, long fluorescence life, large stokes shifts, and sharp linear-like emission band caused by the parityforbidden $\mathrm{f}-\mathrm{f}$ transition. ${ }^{3,8,19-21}$ DPA can transfer energy to lanthanide ions, thus the sensitized lanthanide ions can exhibit characteristic luminescence emission through the antenna effect. ${ }^{21-23}$ Extensive work has been done on lanthanide ion modified carbon dots or quantum dots for the detection of DPA. ${ }^{1-3,6,8}$

Layered rare-earth hydroxide (LRH) is an anionic inorganic layered material, ${ }^{21}$ which is composed of positively charged layers and negatively charged interlayers of anions..$^{20,24-26}$ Due to its rich interlayer chemical properties such as intercalation and ion exchange, it is widely used in the fields of adsorbent, catalysts and photochemical materials. ${ }^{24,27}$ The general formula of LRH is $\left[\mathrm{RE}_{2}(\mathrm{OH})_{5}\left(\mathrm{H}_{2} \mathrm{O}\right) n\right]\left[\mathrm{A}^{m-}\right]_{1 / m}(\mathrm{RE}=$ rare-earth ions, $\mathrm{A}^{m-}=$ anions). ${ }^{24-26,28}$ Delaminated LRH nanosheets would be a very important class of functional 2D nanoscale materials ${ }^{20,29}$ which can be expanded by inserting organic or inorganic ions and further delaminated into monolayer nanosheets. ${ }^{20}$ Investigations have found that 
intercalating surfactant ions such as dodecyl sulfate (SDS) into the interlayer can facilitate the exfoliation and that formamide (FM) is an excellent delaminating reagent. ${ }^{20,30}$ Single luminescence probes based on $\mathrm{Tb}^{3+}$ doped nanosheets have been applied in the detection of $\mathrm{DPA},{ }^{31}$ but the luminescence intensity based on lanthanide ions always suffers from the interference of instrumental factors or microenvironments. Ratiometric luminescence probes are highly attractive due to their self-calibration ability, which can reduce the interference. In this work, a luminescence nanoprobe, $\mathrm{LNP}(\mathrm{Tb})$, was constructed for quantitative luminescence detection of DPA through the intercalation and exfoliation of layered terbium hydroxide $(\mathrm{LTbH})$. It's worth noting that $\operatorname{LNP}(\mathrm{Tb})$ served as a probe because the luminescence intensity of FM remains unchanged as a reference, while the $\mathrm{Tb}^{3+}$ ions can exhibit enhanced sharp luminescence emission upon the sensitization of DPA, enabling ratiometric DPA detection with a linear response in the nanomolar concentration range.

\section{Experimental}

\subsection{Preparation of the Cl-LTbH precursor}

A Cl-LTbH precursor was synthesized by a hydrothermal method as previously reported. ${ }^{20,32}$ An aqueous solution containing $\mathrm{TbCl}_{3} \cdot 6 \mathrm{H}_{2} \mathrm{O}$ (1 $\left.\mathrm{mmol}\right)$, hexamethylenetetramine (HMT) (1 mmol), NaCl (13 mmol), and deionized water (80 $\mathrm{ml}$ ) was heated at $90{ }^{\circ} \mathrm{C}$ for $12 \mathrm{~h}$ in a Teflon-lined autoclave. After the product was filtered, washed, and vacuum-dried (40 ${ }^{\circ} \mathrm{C}$ for $12 \mathrm{~h}$ ), a Cl-LTbH precursor was obtained.

\subsection{Preparation of the SDS-LTbH composite}

The intercalation of SDS into LTbH was carried out by an ion-exchange reaction. ${ }^{20}$ First, $1.26 \mathrm{mmol}$ SDS was added to deionized water to form aqueous solutions. Then the ClLTbH powder $(0.42 \mathrm{mmol})$ was dispersed into the above solutions. Finally, the reaction was conducted at $70{ }^{\circ} \mathrm{C}$ for 48 $\mathrm{h}$ in a Teflon-lined autoclave to give the precipitates, followed by filtration, washing, and vacuum drying. An SDS-LTbH composite was obtained.

\subsection{Exfoliation of the SDS-LTbH composite}

SDS-LTbH (40 mg) was dispersed into formamide $(50 \mathrm{~mL})$, and the resulting colloidal suspension was heated at $40{ }^{\circ} \mathrm{C}$ for 3 days without agitation. The Tyndall effect can be observed, as shown in Fig. 1(D). The prepared luminescence nanoprobe was denoted as $\operatorname{LNP}(\mathrm{Tb})$.

\subsection{DPA detection by LNP(Tb)}

$2.0 \mathrm{~mL}$ of fresh $\mathrm{LNP}(\mathrm{Tb})$ solutions were mixed with $1.0 \mathrm{~mL}$ of fresh DPA solutions with different concentrations (0-100 $\mu \mathrm{M})$. Next, after shaking vigorously for 10 seconds at room temperature, the corresponding luminescence data were measured using a fluorescence spectrophotometer at an excitation wavelength of $285 \mathrm{~nm}$. In particular, all
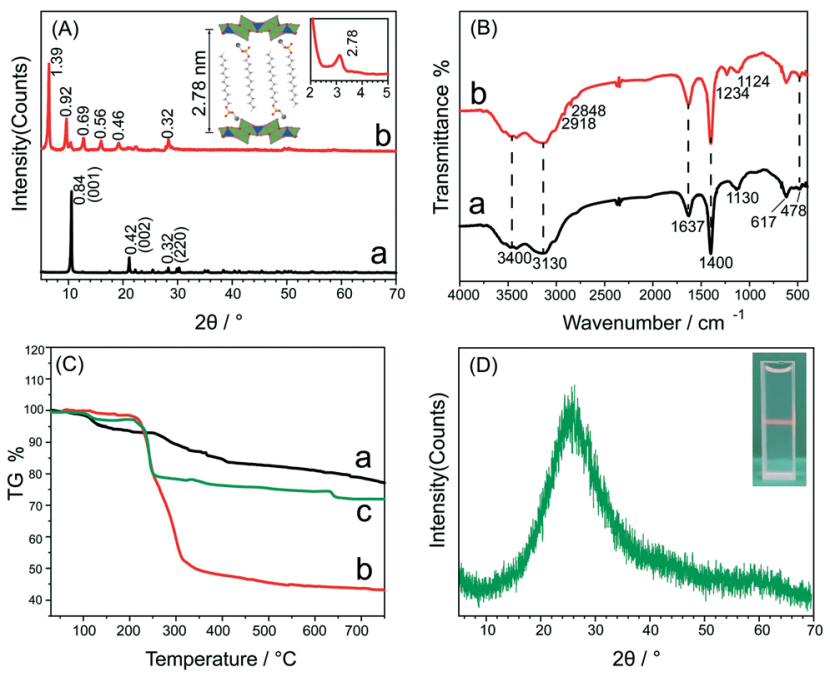

Fig. 1 (A) XRD patterns of the precursor $\mathrm{Cl}-\mathrm{LTbH}$ (a) and the composite SDS-LTbH (b). (B) FT-IR spectra of the precursor Cl-LTbH (a) and the composite SDS-LTbH (b). (C) TG curves of the precursor $\mathrm{Cl}-\mathrm{LTbH}(\mathrm{a}), \mathrm{SDS}$ (b) and the composite SDS-LTbH (c). (D) XRD pattern and the clear Tyndall light scattering of SDS-LTbH colloids.

measurements were carried out in triplicate to measure precision. Selectivity and ion interference experiments were carried out using the same method and concentration (30 $\mu \mathrm{M})$.

\subsection{Characterization}

X-ray diffraction analysis (XRD) was carried out using a Shimadzu7000 X-ray diffractometer equipped with a $\mathrm{Cu}-\mathrm{K} \alpha$ tube and an Al filter $(k=0.1542 \mathrm{~nm})$ generated at $30 \mathrm{~mA}$ and $40 \mathrm{kV}$ in a $2 \theta$ range from 5 to $70^{\circ}$ at a scanning rate of $4^{\circ}$ $\min ^{-1}$. For the small-degree measurements, XRD patterns were obtained in the $2 \theta$ range from 2 to $5^{\circ}$. Fourier transform infrared (FT-IR) spectra were recorded with a VERTEX70 spectrometer. The wavenumber range was set from 4000 to $400 \mathrm{~cm}^{-1}$. The thermal decomposition behavior was measured in flowing air with a TGA HCT-1 thermal analyzer at a heating rate of $10{ }^{\circ} \mathrm{C} \min$ from 30 to $750{ }^{\circ} \mathrm{C}$. The morphologies of the LRH precursor and composite were observed using a field-emission scanning electron microscope (FESEM, Hitachi S-4800 microscope), while energy dispersive X-ray spectroscopy (EDS) mapping was performed on Hitachi S-8010 and Bruker XF lash 6160 instruments. The fluorescence lifetimes of samples were recorded with an Edinburgh FS5 spectrofluorimeter. Luminescence spectra were obtained on a Hitachi F-4500 spectrofluorimeter. The ex/em slit was $5 \mathrm{~nm} / 5 \mathrm{~nm}$, the light source was a xenon lamp, and the voltage was $700 \mathrm{~V}$.

\section{Results and discussion}

\subsection{Characterization of the layered structure}

The XRD patterns of the Cl-LTbH precursor and SDS-LTbH composite are shown in Fig. 1(A). Fig. 1(A)-a shows a series of 
strong (00l) reflections and a basal spacing $\left(d_{\text {basal }}\right)$ of 0.84 $\mathrm{nm}$, and the results for $\mathrm{Cl}-\mathrm{LTbH}$ are consistent with the literature. $^{32}$ The sharp and symmetric diffractions suggest the formation of well-crystallized layered compounds. ${ }^{24,33}$ For SDS-LTbH (Fig. 1(A)-b), a series of sharp and symmetric basal $(00 l)$ reflections are observed at 2.78, 1.39, 0.92, 0.69 and 0.56 $\mathrm{nm}$ with a basal spacing $\left(d_{\text {basal }}\right)$ of $2.78 \mathrm{~nm}$, indicating the well-ordered hydroxide layers. ${ }^{20}$ The difference in the characteristic peaks of the LRH composite and the precursor results from the substitution of SDS anions for $\mathrm{Cl}^{-}$in the interlayer of $\mathrm{LTbH}$. According to previous reports, the thickness of the LRH layer is $0.65 \mathrm{~nm}^{20,28,34}$ and the length of SDS is $1.82 \mathrm{~nm},{ }^{20}$ and an interlayer spacing of $2.13 \mathrm{~nm}$ (= $2.78 \mathrm{~nm}-0.65 \mathrm{~nm}$ ) suggests an alternating antiparallel monolayer arrangement of SDS vertical to the layer, as shown in Fig. 1(A).

The FT-IR spectra of the Cl-LTbH precursor and the SDSLTbH composite are shown in Fig. 1(B). The bands at 3400 $\mathrm{cm}^{-1}$ and $3130 \mathrm{~cm}^{-1}$ belong to the stretching vibration absorptions of interlayer $-\mathrm{OH}$ and interlayer $\mathrm{H}_{2} \mathrm{O}$. The vibration absorption bands at 2918 and $2848 \mathrm{~cm}^{-1}$ are the anti-symmetric and symmetric stretching vibrations in the alkyl chain of SDS, respectively. ${ }^{20}$ The band at $1637 \mathrm{~cm}^{-1}$ indicates the presence of $\mathrm{H}_{2} \mathrm{O}$ molecules as bending vibration. ${ }^{24,25}$ The band at $1400 \mathrm{~cm}^{-1}$ can be assigned to the vibrations of the deprotonated $\mathrm{C}-\mathrm{H}$ group. The absorption band at $1234 \mathrm{~cm}^{-1}$ is the anti-vibration form of $-\mathrm{OSO}_{3}{ }^{-20}$ The bands at 1130 and $1124 \mathrm{~cm}^{-1}$ are ascribed to the $\mathrm{C}-\mathrm{OH}$ stretching vibrations. ${ }^{35,36}$ The band at around $617 \mathrm{~cm}^{-1}$ ascribed to the $\mathrm{Tb}-\mathrm{O}$ vibrations also verify the formation of the layered composites. ${ }^{24,25,28}$

Fig. 1(C) shows the TG results for the precursor, organics, and composite. The weight loss of the composite (Fig. 1(C)-c) is much higher than that of $\mathrm{Cl}-\mathrm{LTbH}$ (Fig. 1(C)-a) but lower than that of SDS (Fig. 1(C)-b), indicating that the composite has a good thermostability.

The XRD pattern of the SDS-LTbH colloids is shown in Fig. 1(D). There are no sharp basal reflections but with a halo in the $2 \theta$ range of $20-30^{\circ}$, which is due to the scattering of liquid formamide. ${ }^{20}$ The XRD result verifies that the composite has been delaminated.
The morphologies of the synthesized samples are depicted in Fig. 2 via SEM observations, which shows a plate-like morphology with a rough surface. It can also be seen that the composites are composed of microcrystalline particles. ${ }^{33} \mathrm{Cl}-$ LTbH (Fig. 2(a)) mainly crystallizes into elongated hexahedral platelets, and some grow into columnar or flower-like aggregates, which are observed in previous studies. ${ }^{24,28,33}$ SDS-LTbH (Fig. 2(b)) keeps the morphology of the Cl-LTbH precursor. A similar morphology between Cl-LTbH and SDS$\mathrm{LTbH}$ reveals topological intercalation during the ion exchange progress. ${ }^{27}$ The elemental mapping images and EDS results in the precursor $\mathrm{Cl}-\mathrm{LTbH}$ and the SDS-LTbH composite are displayed in Fig. 2, which show the presence of different layer elements and the interlayer organic species. The relative brightness of the elements represents their corresponding content.

\subsection{Luminescence properties of FM and LNP(Tb)}

As shown in Fig. 3, the excitation spectrum of LNP(Tb) (Fig. 3(c)) was explored at an emission wavelength of $332 \mathrm{~nm}$, and the emission spectrum of FM (Fig. 3(a)) and LNP(Tb) (Fig. 3(b)) was explored at an excitation wavelength of $285 \mathrm{~nm}$, respectively. The emission at $332 \mathrm{~nm}$ belongs to FM, which acts as a reference in the detection. There exist weak $\mathrm{Tb}^{3+}$ characteristic emissions and a strong double frequency scattering peak (570 $\mathrm{nm})$.

\subsection{The detection of DPA}

A quantitative luminescence study was conducted to evaluate the sensitivity of the ratiometric luminescence nanoprobe for DPA. Fig. 4(A) shows the luminescence response $\left(\lambda_{\mathrm{ex}}=285\right.$ $\mathrm{nm}$ ) of the nanoprobe when introducing different concentrations of DPA into the system. The emission spectrum of the nanoprobe without DPA was dominated by $\mathrm{FM}$, with the major emission peak at $332 \mathrm{~nm}$. Due to the antenna effect, ${ }^{22,23}$ efficient energy transfers from DPA to $\mathrm{Tb}^{3+}$ ions, and the addition of a small amount of aqueous DPA solution enhances the emission intensity of $\mathrm{Tb}^{3+}$ ions. The emission peaks at 490, 544, 582, and $619 \mathrm{~nm}$ are attributed to the characteristic transitions of $\mathrm{Tb}^{3+}$ ions, from
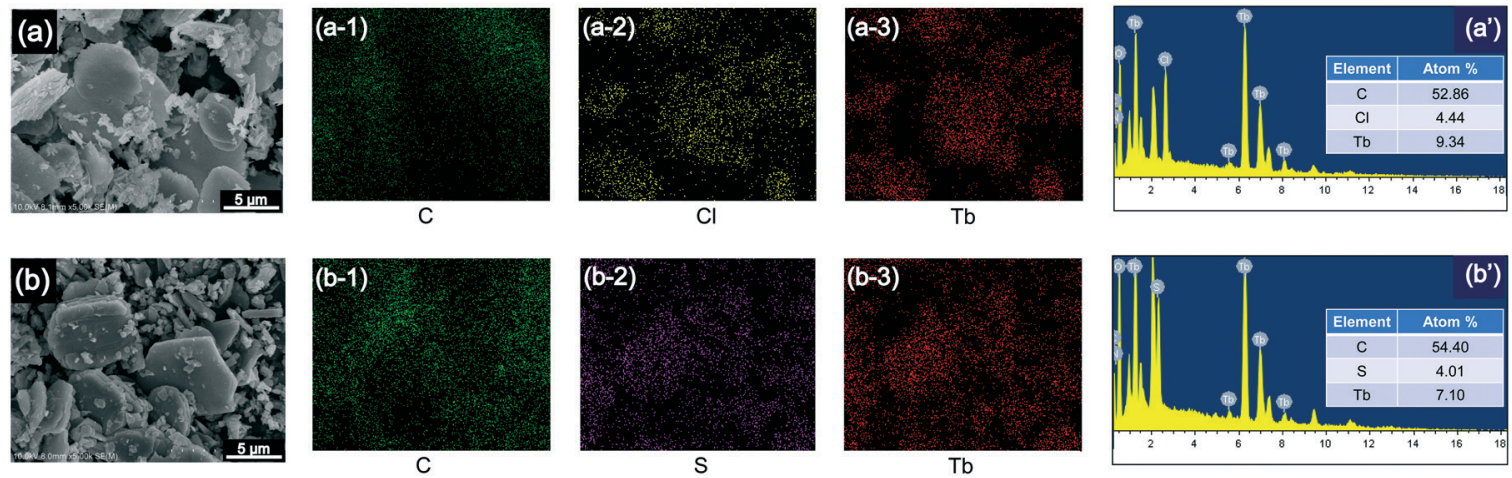

Fig. 2 SEM images of Cl-LTbH (a) and SDS-LTbH (b) and their corresponding elemental distribution mapping images ((a-1)-(a-3) and (b-1)-(b-3)) and related EDS results (a' and b'). 


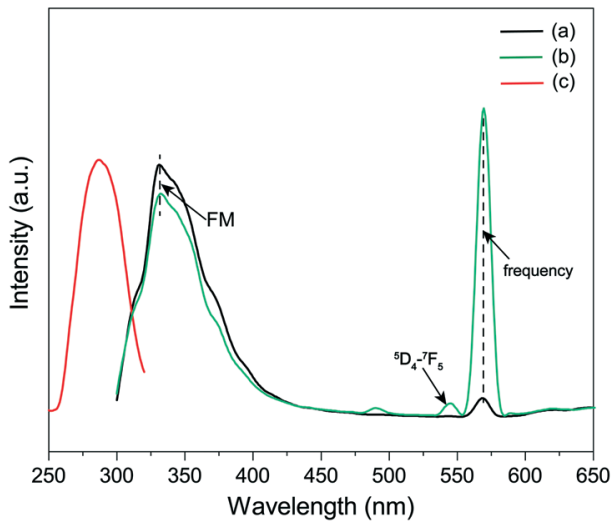

Fig. 3 Emission spectrum of FM ( $\lambda_{\text {ex }}=285 \mathrm{~nm}$ ) (a). Emission spectrum of $\operatorname{LNP}(\mathrm{Tb})\left(\lambda_{\mathrm{ex}}=285 \mathrm{~nm}\right)$ (b). Excitation spectrum of $\operatorname{LNP}(\mathrm{Tb})\left(\lambda_{\mathrm{em}}=\right.$ $332 \mathrm{~nm})(\mathrm{c})$.

the ${ }^{5} \mathrm{D}_{4}$ energy level to ${ }^{7} \mathrm{~F}_{J}$ multiplets $(J=6,5,4$, and 3$)$, respectively. ${ }^{5,6,8,20}$ The maximum emission peak at $544 \mathrm{~nm}$ is narrow and sharp, thus resulting in excellent ratiometric measurements of DPA concentration. ${ }^{8}$ The FL intensity of $\mathrm{Tb}^{3+}$ increases with the DPA concentration, especially for the emission at $544 \mathrm{~nm}$. Furthermore, the FL intensity of FM at $332 \mathrm{~nm}$ remains constant with the addition of DPA, which can act as a ratiometric luminescence nanoprobe to detect DPA. According to Fig. 4 (B), the FL intensity ratio $\left(I_{544} / I_{332}\right)$ of $\operatorname{LNP}(\mathrm{Tb})$ is proportional to the concentration of DPA in the range of $1-100 \mu \mathrm{M}$ and exhibits a strong linear relationship
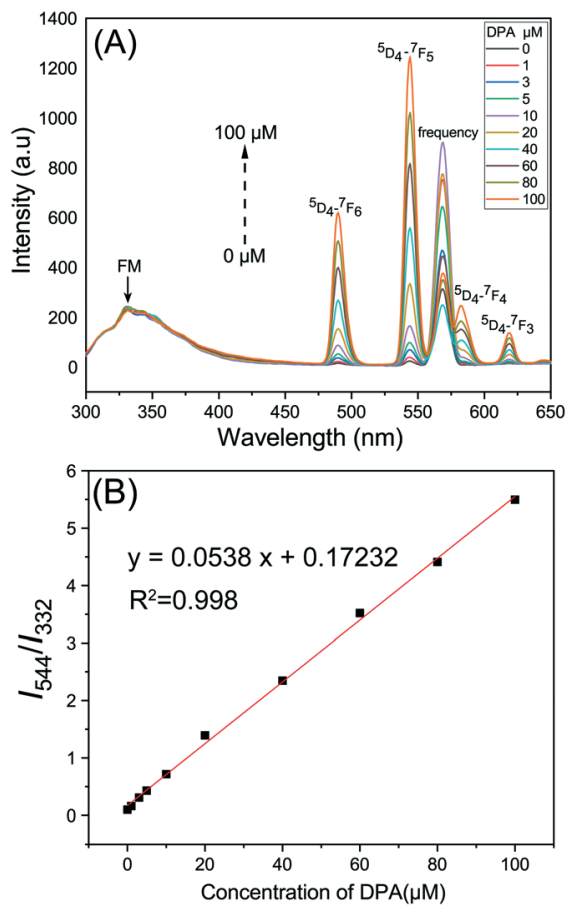

Fig. 4 (A) Evolution of the luminescence spectra of $L N P(T b)$ on increasing the DPA concentration $\left(\lambda_{\mathrm{ex}}=285 \mathrm{~nm}\right)$. (B) Linear relationship between the DPA concentration and the fluorescence ratio $\left(I_{544} / /_{332}\right)$ of $\mathrm{LNP}(\mathrm{Tb})$ in the concentration range of $1-100 \mu \mathrm{M}$. of $R^{2}=0.998$. The limit of detection (LOD) was determined to be $36.67 \mathrm{nM}\left(\mathrm{LOD}=3 S_{\mathrm{b}} / m, m\right.$ is the slope of the calibration curve and $S_{\mathrm{b}}$ is the standard deviation of the blank), which is three orders of magnitude lower than an infectious dosage of the Bacillus thoracic spores $\left(6 \times 10^{-5} \mathrm{M}\right.$ required $){ }^{8,37} \mathrm{~A}$ comparison with a previously reported lanthanide based probe is shown in Table S1, $\dagger$ which shows that the probe has a relatively low detection limit and a wider detection range.

\subsection{Interference detection}

To study the selectivity of the system as a luminescence nanoprobe for DPA detection, the $\operatorname{LNP}(\mathrm{Tb})$ responses toward various potentially interfering substances are investigated, including aromatic carboxylic acids, amino acids and common biorelevant ions that are either structurally related to or can potentially be present in many bacterial spores along with DPA. ${ }^{8}$

As shown in Fig. 5, the selected interfering substances include glutamic (Glu), glycine (Gly), aspartic (Asp), phenylalanine (Phe), formic (FA), benzoic (BA), $m$-phthalic $(m$-PA) and $m$-toluic $(m$-TA) acid, which can coordinate with $\mathrm{Tb}^{3+}$ ions. In addition, common biorelevant ions $\left(\mathrm{Cl}^{-}, \mathrm{K}^{+}\right.$, $\mathrm{Fe}^{3+}, \mathrm{Ca}^{2+}, \mathrm{Mg}^{2+}, \mathrm{Na}^{+}, \mathrm{Cu}^{2+}, \mathrm{Al}^{3+}$, and $\left.\mathrm{Ag}^{+}\right)$are tested. Remarkably, the FL intensity ratio $\left(I_{544} / I_{332}\right)$ of the nanoprobe is almost unchanged when the interfering substance is added alone. However, when DPA is added, the FL intensity ratio $\left(I_{544} / I_{332}\right)$ of the nanoprobes is significantly enhanced. It can
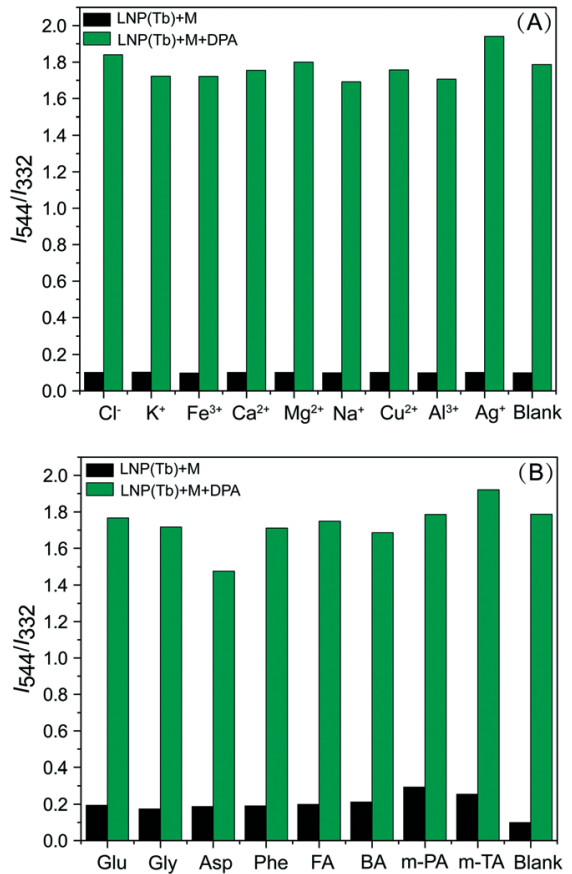

Fig. 5 Luminescence responses of LNP(Tb) toward different potentially interfering aromatic carboxylic acids or amino acids (A) and common biorelevant ions (B). The black bars represent an addition of $30 \mu \mathrm{M}$ of the interfering acid or ion to $\operatorname{LNP}(\mathrm{Tb})$. The green bars represent a subsequent addition of $30 \mu \mathrm{M}$ DPA to the solution $\left(\lambda_{\mathrm{ex}}=285 \mathrm{~nm}\right)$. 
be concluded that only DPA can induce significant luminescence enhancement at the same concentration, while the luminescence changes caused by other interference are negligible. Due to its excellent selectivity and antiinterference ability, the ratiometric sensing capability of this nanoprobe is not only useful for the quantitative determination of the DPA concentration but also not affected by intensity fluctuations due to instrumental or environmental factors. So this luminescence nanoprobe has great potential to be used for detecting DPA in complex samples.

\subsection{Sensing applications in tap water}

The usefulness of $\operatorname{LNP}(\mathrm{Tb})$ was evaluated by adding different concentrations of DPA to the tap water samples. As listed in Table 1, the experimental results show a good recovery rate and small relative standard deviation (RSD), and the recoveries of DPA are in the range of 96.65-101.79\% with an RSD of $0.13-1.91 \%$ for tap water samples. This indicates that most of the results are accurate and reliable. As a result, the prepared $\mathrm{LNP}(\mathrm{Tb})$ can detect DPA in tap water samples accurately.

\subsection{Fluorescence lifetimes of LNP(Tb)}

As shown in Fig. 6, to further explore the antenna effect behavior between DPA and $\mathrm{Tb}^{3+}$, fluorescence lifetime is measured at an excitation wavelength of $285 \mathrm{~nm}$ and collected at an emission wavelength of $544 \mathrm{~nm}$. The measurement samples are the $\operatorname{LNP}(\mathrm{Tb})$ with different concentrations of DPA $(0 \mu \mathrm{M}$ and $30 \mu \mathrm{M})$. The logarithmic decay graphs are fitted with the single-exponential equation $I(t)=I_{0} \exp (-t / \tau)$, where $I(t)$ is the fluorescence intensity dependent on time, $I_{0}$ is the fluorescence intensity at time zero, and $\tau$ is the lifetime. ${ }^{27} \operatorname{LNP}(\mathrm{Tb})$ with a concentration of $30 \mu \mathrm{M}$ DPA $(\tau=0.85 \mathrm{~s})$ shows a longer fluorescence lifetime than $\operatorname{LNP}(\mathrm{Tb})$ without DPA $(\tau=0.69 \mathrm{~s})$. The results can be attributed to the antenna effect between DPA and $\mathrm{Tb}^{3+}$, which enhances the luminescence of $\mathrm{Tb}^{3+}$ through energy transfer.

\subsection{Luminescence mechanism analysis}

Normally, the sensitization luminescence effect needs an effective energy transfer from the activators to the acceptors, ${ }^{33,34}$ which means a perfect fit of the energy gap between the excited state energy levels of $\mathrm{Tb}^{3+}$ and the triplet state energy levels of the organics. If the energy difference is too large, then the probability of electron conversion will be little, thus decreasing the chance of transition. If the energy difference is too small, inverse or mutual transition may

Table 1 Detection of DPA in real samples $(n=3)$ by the probe

\begin{tabular}{lcccl}
\hline Sample & Added $(\mu \mathrm{M})$ & Found $(\mu \mathrm{M})$ & Recovery $(\%)$ & RSD (\%) \\
\hline Tap water & 5.0 & 4.83 & 96.65 & 1.07 \\
& 10.0 & 10.17 & 101.79 & 1.91 \\
& 20.0 & 20.21 & 101.08 & 0.13
\end{tabular}
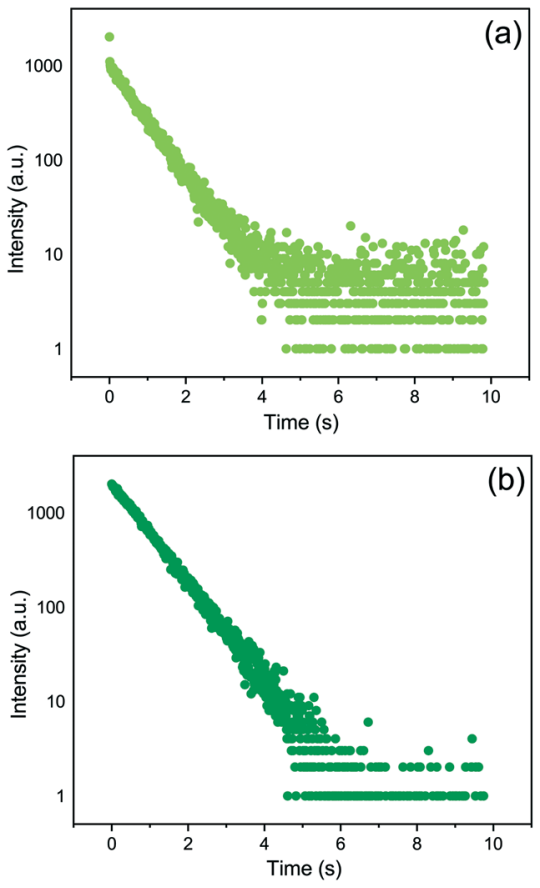

Fig. 6 Photoluminescence decay curves of LNP(Tb) without DPA (a) and with $30 \mu \mathrm{M}$ DPA (b) (excited at $285 \mathrm{~nm}$ and monitored at $544 \mathrm{~nm}$ ).

occur, while most of the energy would be inactivated by vibration rather than luminescence. ${ }^{33}$ An energy gap of 3500 $\mathrm{cm}^{-1}$ or higher is necessary to facilitate efficient and irreversible energy transfer. ${ }^{23,33}$ As shown in Scheme 1, the mechanism of the energy transfer can be ascribed to the antenna effect: DPA is excited by energy (usually the ultraviolet light) and electrons generate the $\pi-\pi$ transition. That is, electrons transfer from the ground state to an excited singlet state $\left(S_{0} \rightarrow S_{1}\right)$, then quickly transfer from excited singlet state to excited triplet state $\left(S_{1} \rightarrow T_{1}\right)$ via intersystem crossing. Next, DPA at $T_{1}$ transfer energy to the lowest excited state of $\mathrm{Tb}^{3+}$, thus emitting the characteristic luminescence of $\mathrm{Tb}^{3+} \cdot{ }^{38,39}$ The energy gap between the triplet energy level of DPA $\left(25510 \mathrm{~cm}^{-1}\right)$ and the ${ }^{5} \mathrm{D}_{4}$ level of $\mathrm{Tb}^{3+}\left(20540 \mathrm{~cm}^{-1}\right)^{23}$ is $4970 \mathrm{~cm}^{-1}$, which is an excellent effective energy transfer.

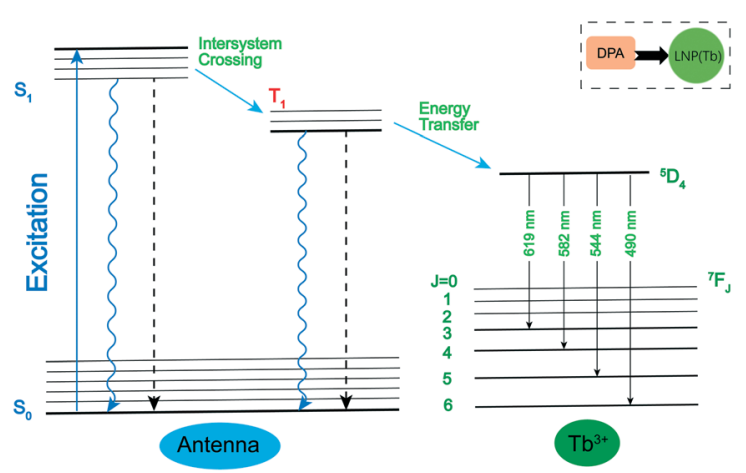

Scheme 1 Schematic diagram of the antenna effect mechanism of $\mathrm{Tb}^{3+}$. 
As a result, DPA can sensitize $\mathrm{Tb}^{3+}$ luminescence effectively, which provides a viable method to detect DPA.

\section{Conclusions}

In summary, a ratiometric luminescence nanoprobe was prepared by a simple method for quantitative detection of DPA. The XRD and FT-IR results indicate that SDS is successfully intercalated into LTbH to form an SDS-LTbH composite. Based on the Tyndall effect and XRD pattern of the SDS-LTbH colloids, SDS-LTbH has been successfully delaminated into nanosheets in FM. In the concentration range of $1-100 \mu \mathrm{M}$, the DPA concentration is linearly proportional to the FL intensity of $\mathrm{Tb}^{3+}$ at $544 \mathrm{~nm}$. However, the FL intensity of FM at $332 \mathrm{~nm}$ remains constant, thus enabling the ratiometric detection of DPA. In addition, the luminescence nanoprobe exhibits a highly selective and sensitive detection of DPA, with a detection limit of $36.67 \mathrm{nM}$ and long-term photostability. It's worth noting that LNP(Tb) can also be applied to detect DPA in tap water samples with good recovery rates and small RSD. This work provides a novel and effective method for quantitative detection of an anthrax biomarker.

\section{Conflicts of interest}

There are no conflicts to declare.

\section{Acknowledgements}

This work is supported by the Scientific Research Project of Beijing Educational Committee (KM202110017008), the Youth Top-notch Talent Training Program for Universities and Colleges under Beijing Municipality (CIT\&TCD201804035), and the National Natural Science Foundation of China (21601016).

\section{Notes and references}

1 H. Yang, F. Lu, X. Zhan, M. Tian, Z. Yuan and C. Lu, A $\mathrm{Eu}(3+)$-inspired fluorescent carbon nanodot probe for the sensitive visualization of anthrax biomarker by integrating EDTA chelation, Talanta, 2020, 208, 120368-120376.

2 X. Liu, D. Chen, C. Wang, N. Tian, Z. Li, Y. Zhang and Z. J. Ding, A turn-on luminescence probe for highly selective detection of an anthrax biomarker, Luminescence, 2020, 35, 601-607.

3 J. Wang, D. Li, Y. Qiu, X. Liu, L. Huang, H. Wen and J. $\mathrm{Hu}$, An europium functionalized carbon dot-based fluorescence test paper for visual and quantitative point-ofcare testing of anthrax biomarker, Talanta, 2020, 220, 121377-121384.

4 B. Ma, F. Zeng, F. Zheng and S. Wu, Fluorescent detection of an anthrax biomarker based on PVA film, Analyst, 2011, 136, 3649-3655.

5 X. Zhang, W. Zhang, G. Li, Q. Liu, Y. Xu and X. Liu, A ratiometric fluorescent probe for determination of the anthrax biomarker 2,6-pyridinedicarboxylic acid based on a terbium(III)- functionalized UIO-67 metal-organic framework, Microchim. Acta, 2020, 187, 122-129.

6 Y. Y. Ma, Z. J. Wang and D. J. Qian, Ratiometric fluorescence detection of anthrax biomarker based on terbium (III) functionalized graphitic carbon nitride nanosheets, Talanta, 2021, 230, 122311-122319.

7 J. Kim, H. Kim, N. Lee, T. Kim, H. Kim, T. Yu, I. Song, W. Moon and T. Hyeon, Multifunctional Uniform Nanoparticles Composed of a Magnetite Nanocrystal Core and a Mesoporous Silica Shell for Magnetic Resonance and Fluorescence Imaging and for Drug Delivery, Angew. Chem., Int. Ed., 2008, 47, 8438-8441.

8 H. Chen, Y. Xie, A. M. Kirillov, L. Liu, M. Yu, W. Liu and Y. Tang, A ratiometric fluorescent nanoprobe based on terbium functionalized carbon dots for highly sensitive detection of an anthrax biomarker, Chem. Commun., 2015, 51, 5036-5039.

9 K. Ai, B. Zhang and L. Lu, Europium-based fluorescence nanoparticle sensor for rapid and ultrasensitive detection of an anthrax biomarker, Angew. Chem., Int. Ed., 2009, 48, 304-308.

10 M. L. Cable, J. P. Kirby, K. Sorasaenee, H. Gray and A. Ponce, Bacterial Spore Detection by $[\mathrm{Tb}(3+)($ macrocycle)(dipicolinate)] Luminescence, J. Am. Chem. Soc., 2007, 129, 1474-1475.

11 L. Meng, A. Bbc, H. Jia, B. Cml, F. Yuan and Z. Cheng, Anthrax biomarker: An ultrasensitive fluorescent ratiometry of dipicolinic acid by using terbium(III)-modified carbon dots, Talanta, 2019, 191, 443-448.

12 N. Dey, D. Biswakarma and S. Bhattacharya, Metal Complex as an Optical Sensing Platform for Rapid Multimodal Recognition of a Pathogenic Biomarker in Real-Life Samples, ACS Sustainable Chem. Eng., 2018, 7, 569-577.

13 M. Yilmaz and H. A. Oktem, Eriochrome Black T-Eu ${ }^{3+}$ complex as a ratiometric colorimetric and fluorescent probe for the detection of dipicolinic acid, a biomarker of bacterial spores, Anal. Chem., 2018, 90, 4221-4225.

14 Y. Luo, L. Zhang, L. Zhang, B. Yu, Y. Wang and W. Zhang, Multiporous Terbium Phosphonate Coordination Polymers Microspheres as Fluorescent Probe for Trace Anthrax Biomarker Detection, ACS Appl. Mater. Interfaces, 2019, 11, 15998-16005.

15 X. Bai, Y. Zeng, X. Zhou, X. Wang, A. G. Shen and J. Hu, Environmentally Safe Mercury(II) Ions Aided ZeroBackground and Ultrasensitive SERS Detection of Dipicolinic Acid, Anal. Chem., 2017, 89, 10335-10342.

16 J. Fichtel, H. Sass and J. Rullkötter, Assessment of spore contamination in pepper by determination of dipicolinic acid with a highly sensitive HPLC approach, Food Control, 2008, 19, 1006-1010.

17 K. M. Y. Yoon, Recent advances in rapid and ultrasensitive biosensors for infectious agents: lesson from Bacillus anthracis diagnostic sensors, Analyst, 2010, 135, 1182-1190.

18 R. M. Jarvis and R. Goodacre, Discrimination of Bacteria Using Surface-Enhanced Raman Spectroscopy, Anal. Chem., 2004, 76, 40-47. 
19 R. Ju and Q. Gu, Biohybrid based on layered terbium hydroxide and applications as drug carrier and biological fluorescence probe, Appl. Organomet. Chem., 2018, 32, 3926-3934.

20 Y. Zhao, J. G. Li, M. Guo and X. Yang, Structural and photoluminescent investigation of $\mathrm{LTbH} / \mathrm{LEuH}$ nanosheets and their color-tunable colloidal hybrids, J. Mater. Chem. C, 2013, 1, 3584-3592.

21 C. Tan, Q. Wang and C. C. Zhang, Optical and electrochemical responses of an anthrax biomarker based on single-walled carbon nanotubes covalently loaded with terbium complexes, Chem. Commun., 2011, 47, 12521-12523.

22 M. C. Heffern, L. M. Matosziuk and T. J. Meade, Lanthanide probes for bioresponsive imaging, Chem. Rev., 2014, 114, 4496-4539.

23 Y. Zhou, X. Li, L. Zhang, Y. Guo and Z. Shi, 3-D silver(I)lanthanide(III) heterometallic-organic frameworks constructed from 2,2'-bipyridine-3,3'-dicarboxylic acid: synthesis, structure, photoluminescence, and their remarkable thermostability, Inorg. Chem., 2014, 53, 3362-3370

24 Q. Gu, J. Li, L. Ji, R. Ju, H. Jin and R. Zhang, Fabrication of novel bifunctional nanohybrid based on layered rare-earth hydroxide with magnetic and fluorescent properties, Front. Mater. Sci., 2020, 14, 488-496.

25 Q. Gu, Y. Wu and S. Yu, Organic-inorganic luminescent composites obtained by the intercalation of organic dyes into the layered rare-earth hydroxides, Inorg. Chim. Acta, 2019, 487, 162-168.

26 Q. Gu, M. Yuan, S. Ma and G. Sun, Structures and photoluminescence properties of organic-inorganic hybrid materials based on layered rare-earth hydroxides, J. Lumin., 2017, 192, 1211-1219.

27 R. Guo, J. Li, L. Chen, Z. Yu, H. Yao, K. Shi, C. Li and S. Ma, SDC/OS-LDH composite for highly sensitive fluorescence detection of $\mathrm{Fe}(3+)$ at a much lower concentration, Dalton Trans., 2020, 49, 10413-10420.

28 Q. Gu, W. Chen, F. Duan and R. Ju, Fabrication of a nanodrug delivery system based on layered rare-earth hydroxides integrating drug-loading and fluorescence properties, Dalton Trans., 2016, 45, 12137-12143.

29 Q. Gu, F. Su, L. Ma, S. Ma, G. Sun and X. Yang, Intercalation of coumaric acids into layered rare-earth hydroxides: controllable structure and photoluminescence properties, J. Mater. Chem. C, 2015, 3, 4742-4750.

30 L. Hu, R. Ma, T. Ozawa and T. Sasaki, Exfoliation of Layered Europium Hydroxide into Unilamellar Nanosheets, Chem. Asian J., 2010, 5, 248-251.

31 B. Wang, J. Xia, G. Zhou, X. Li, M. Dai, D. Jiang and Q. Li, $\mathrm{Tb}$ (III)-doped nanosheets as a fluorescent probe for the detection of dipicolinic acid, RSC Adv., 2020, 10, 37500-37506.

32 F. Su, Q. Gu, S. Ma, G. Sun, X. Yang and L.-D. Zhao, Delaminated layered rare-earth hydroxide composites with ortho-coumaric acid: color-tunable luminescence and blue emission due to energy transfer, J. Mater. Chem. C, 2015, 3, 7143-7152.

33 R. Guo, F. Su, H. Wang, Y. Guo, H. Yao, G. Huang, J. Li, Z. Liang, K. Shi and S. Ma, Luminescence Tuning of Layered Rare-Earth Hydroxides (LRHs, R = Tb, Y) Composites with 3-Hydroxy-2-naphthoic Acid and Application to the Fluorescent Detection of $\mathrm{Al}(3+)$, Inorg. Chem., 2019, 58, 4979-4988.

34 N. Chu, Y. Sun, Y. Zhao, X. Li and X. Yang, Intercalation of organic sensitisers into layered europium hydroxide and enhanced luminescence property, Dalton Trans., 2012, 41, 7409-7414.

35 M. Luo, S. Xu, Q. Gu, Z. Di, Q. Liu and Z. Zhao, Co-Al nanosheets derived from LDHs and their catalytic performance for syngas conversion, J. Colloid Interface Sci., 2019, 538, 440-448.

36 Y. Zhu, Y. Zhou, Z. Tao, M. He, Y. Wang, X. Yang and Y. Yong, Preparation and characterization of lactateintercalated Co-Fe layered double hydroxides and exfoliated nanosheet film with low infrared emissivity, Appl. Surf. Sci., 2012, 263, 132-138.

37 P. M. Pellegrino, N. F. Fell, D. L. Rosen and J. B. Gillespie, Bacterial Endospore Detection Using Terbium Dipicolinate Photoluminescence in the Presence of Chemical and Biological Materials, Anal. Chem., 1998, 70, 1755-1760.

38 E. G. Moore, A. P. S. Samuel and K. N. Raymond, From Antenna to Assay: Lessons Learned in Lanthanide Luminescence, Acc. Chem. Res., 2009, 42, 542-552.

39 C. P. Montgomery, B. S. Murray, E. J. New, R. Pal and D. Parker, Cell-Penetrating Metal Complex Optical Probes: Targeted and Responsive Systems Based on Lanthanide Luminescence, Acc. Chem. Res., 2009, 42, 925-937. 